\title{
Fractured Tracheostomy Tube- An Unusual Foreign Body in Tracheobronchial Tree
}

\author{
Sanu P Moideen, ${ }^{1}$ G Arun, ${ }^{1}$ M Mohan, ${ }^{1}$ Khizer Hussain Afroze $e^{2}$
}

\section{Introduction}

ABSTRACT

Approximately $20 \%$ of patients with tracheostomy are discharged from hospital with the tracheostomy tube in situ. Proper long term care and management of such tracheostomy patients remains as a challenge to care givers. Fracture of metallic tracheostomy tube (TT) with aspiration of the fragment into tracheobronchial airway is a rare complication of tracheostomy. Case Report

Here we are presenting a case of a 42-year-old male patient, presented to the emergency department with complaint of mild respiratory distress following aspiration of fractured metallic TT and a novel method in removing the dislodged fragment.

Discussion

A detailed review of literature has been included to discuss different aspects of aspiration of fractured fragment of tracheostomy tube and best practice recommendations for proper tracheostomy care.

Conclusion

Educating the care-giver about care of the tracheostomized patient in general and care of the tracheostomy tube in particular, may help reduce accidental complications.

Kevwords

Tracheostomy; Foreign bodies

$\mathrm{T}$ racheostomy is a common procedure usually performed as part of modern airway management in many specialties like surgical, medical and critical care units. Globally the number of tracheostomies being performed is increasing ${ }^{1}$ due to many reasons like cancer of larynx, nasopharynx, severe head injury, spinal cord injury, vocal cord paralysis motor neuron disease etc. Approximately $20 \%$ of tracheostomy patients are discharged from hospital with the tracheostomy tube insitu. ${ }^{2,3}$ These patients with permanent tracheostomies need proper long term care and management. Inappropriate care can result in unexpected adverse outcomes.

Fracture of metallic tracheostomy tube (TT) with aspiration of the fragment into tracheobronchial airway is a rare complication of tracheostomy. In extreme cases, this can fail the whole purpose of tracheostomy by compromising the airway, which necessitates urgent medical attention. Hence there is a need to understand this particular complication, for prevention, early recognition and prompt management.

Here we present a case of a 42 year old patient, who attended the emergency department with the complaint of mild respiratory distress following aspiration of fractured metallic TT and showcase a novel method for the extraction of the same. We also include a review of literature and best practice recommendations for proper tracheostomy care.

\section{Case Report}

A 42-year-old male patient presented to emergency department with complaint of mild respiratory distress

1 - Department of ENT \& HNS, Sri Siddhartha Medical

College \& Hospital, Tumkur, Karnataka

2 - Department of Anatomy, Sri Siddhartha Medical College \& Hospital, Tumkur, Karnataka

Corresponding author:

Dr Sanu P Moideen

email: drsanu85@gmail.com 
following aspiration of a broken tracheostomy tube. This happened while his wife was attempting the removal of tube for routine cleaning. She noticed that the tube part was missing and only neck plate (shield) was in place. The patient has undergone tracheostomy 3 years back due to bilateral abductor palsy following total thyroidectomy for papillary carcinoma thyroid in another institute. For last 2 years, he never had follow up with the hospital where he was initially treated and continued to use the metallic tube for two years.

At time of presentation in emergency room (ER) the patient was hemodynamically stable with mild tachypnea (respiratory rate was 22 /minute). His oxygen saturation was $97 \%$ on air. Neck examination revealed a patent stoma. On auscultation air entry was reduced on left side of chest. Rhonchi was present. A chest X-ray (postero-anterior view) revealed fractured TT in left main bronchus.(Fig. 1) He was given oxygen inhalation, nebulization and steroids in the ER and then shifted to operating room (OR).

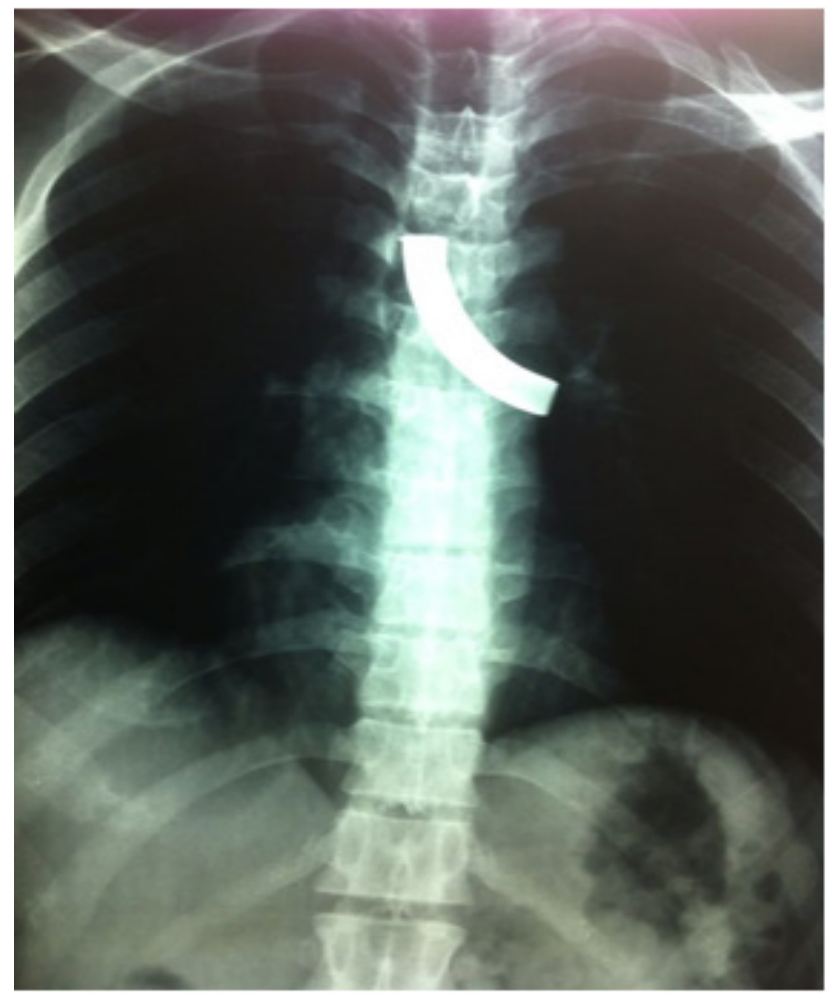

Fig. 1. Chest Xray showing fractured and aspirated tracheostomy tube in left main bronchus
In the OR, intravenous sedation was given. Patient was pre-oxygenated through the stoma. Under apneaoxygenation-apnea technique a $0^{\circ} 4 \mathrm{~mm}$ Storz ${ }^{\circledR}$ nasal endoscope connected to camera and monitor was passed through the stoma with minimal difficulty. The broken fragment was visualized in lower trachea and left main bronchus. The fragment was gently dislodged with bronchoscope forceps and then withdrawn slowly along with the endoscope. The patient was oxygenated again to maintain the saturation. The scope passed again to visualize the tracheal lumen. The tracheal mucosa was appearing to be congested and edematous. After removal of the broken fragment, a new PVC tube ( 8 $\mathrm{mm}$ ) was inserted and secured into the tracheostomy site. Inspection of the fractured piece showed severe corrosion at junction between tube and neck plate.

The patient was observed for 12 hours in the intensive care unit (ICU) and was then shifted to ward. The postoperative period was uneventful. He was discharged on next day with instructions for regular follow up and was educated regarding tracheostomy care.

\section{Discussion}

The first case of aspiration of fractured tracheostomy tube was reported by Bassoe and Boe in 1960. ${ }^{4}$ Since then, similar cases are reported in literature from time to time. The largest case series was reported by Gupta et al in 1987 , over a period of 8 years consisting 9 patients..$^{5}$

Fracture and aspiration of TT have been reported in all age groups, with various tube materials including silver, stainless steel, polyvinyl chloride (PVC) and silicone. Piromachai et al, in their review of 20 cases from 18 published reports described fracture TT in 15 males $(75 \%)$ and four females $(20 \%)$. Fourteen cases were fracture of metallic tube, while three cases were PVC tubes. ${ }^{6}$ In their review, the most common sites of lodgment were the trachea and right main bronchus.

In most of the cases, the fracture site was the junction between tube and neck plate. Fracture at distal end and fenestra are also reported. ${ }^{6}$ The possible reasons for fracture is thought to be progressive weakening due to repeated boiling and sterilizing, ${ }^{5,7}$ especially with use of bleaching powder. ${ }^{8}$ Jensen et al proposed mechanical 
stress due to repeated use is likely to be greatest at tubeflange junction area. ${ }^{9}$ Okafor reported that stenosis of the tracheal stoma can increase the risk of fracture. ${ }^{10}$ Other reasons postulated are due to aging of the tube, manufacturing defects or due to poor quality of the TT. Alkaline bronchial secretions accumulating around the TT can also corrode the tube. ${ }^{11}$

So-ngern and Boonsarngsuk in their series reported that tracheostomy tube fracture can happen any time between 5 days to 22 years after the placement. ${ }^{12}$ Karakoc et al reported that, patients with a fractured tracheostomy tube can present within a duration of 1 day -132 days, with a median interval of three months. ${ }^{13}$ The presentation is most often with mild respiratory distress. ${ }^{9}$ Other symptoms are cough, hemoptysis, wheezing, recurrent pneumonia, difficulty in suction or re-insertion of inner tube. Death also is reported, especially in pediatric population probably due to the small airway caliber. ${ }^{8,14}$ The most common dislodged site of fractured TT is right main bronchus. This is because right main bronchus is consistently steeper and slightly wider. ${ }^{15}$ There are other case reports involving left main bronchus, carina, left posterior basal segment also. ${ }^{6}$ In our case, the fractured segment was located in distal end of trachea and left main bronchus.

The diagnosis can be made from history, clinical examination and with a plain x-ray of chest (posteroanterior view). Once diagnosed, primary care should be given to the patient. Foreign body aspiration is a medical emergency and prompt action is needed. ${ }^{16}$ Rigid bronchoscopy under controlled environment like operation theatre is the recommended option for removal of large foreign bodies in literatures. Some authors have reported need for thoracotomy and bronchotomy also. ${ }^{17}$ In this case we followed a novel method for removal of the fractured segment, by using a rigid $0^{\circ}$ nasal endoscope under apnea-oxygenation technique.

Majority of case reports on aspiration of fractured TT is from developing countries. This may be because of continuous use of the same tube for prolonged periods due to economic constraints associated with replacing tubes or use of inferior quality tubes, loss in follow up, poor tracheostomy care etc. ${ }^{18}$ Proper tracheostomy care is the most important step in prevention of this complication. There exists no consensus for proper tracheostomy care.
From our experience and previous results, we are put forwarding following recommendations.

1. Planned discharge and ongoing support is essential for tracheostomy patients. ${ }^{2}$

2. Proper training and guidelines are needed for community practitioners, general practitioners, nurses and paramedics on discharge and care of patients with tracheostomy, especially in remote areas.

3. The tube should be checked for any manufacturing defects or signs of wear and tear before inserting. ${ }^{17}$

4. Regular follow up and twice yearly replacement of tracheostomy tube. ${ }^{19,20}$

5. Regular checking of tube for wears and tears.

6. Clean the inner cannula daily, every other day or more frequently as needed..$^{19,20}$

7. Daily changing of dressings at tracheostomy site. ${ }^{20}$

8. Weekly replacement of tube ties..$^{20}$

9. Proper education of patient and caregiver about tracheostomy care is most important.

10. In case of an aspiration, early identification and intervention with rigid bronchoscopic removal under controlled environment is recommended..$^{18}$

\section{Conclusion}

Fracture and aspiration of tracheostomy tube is a rare complication which requires prompt diagnosis and immediate intervention. Patient and care giver education of tracheostomy tube maintenance, regular follow up, timely replacement of tracheostomy tube etc. are important for preventing such complications.

\section{References}

1. Parker V, Shylan G, Archer W, McMullen P, Smith K, Giles M, Morrison J, Austin N. Trends and challenges in the management of tracheostomy in older people: the need for a multidisciplinary team approach. Contemporary nurse 2007; 26(2):177-83

2. Everitt E. Care of patients with permanent tracheostomy. Nursing times 2016; 112(21-23):20

3. Lewis CW, Carron JD, Perkins JA, Sie KC, Feudtner C. Tracheotomy in pediatric patients: a national perspective. Arch Otolaryngol Head Neck Surg. 2003; 129(5):523-9

4. Bassoe HH, Bde J. Broken tracheotomy tube as a foreign body. The Lancet; 275(7132):1006-7

5. Gupta SC. Fractured tracheostomy tubes in the tracheobronchial tree: A report of nine cases. J Laryngol Otol. 1987; 101(08):861- 
7

6. Piromchai $P$, Lertchanaruengrit $P$, Vatanasapt $P$, Ratanaanekchai T, Thanaviratananich S. Fractured metallic tracheostomy tube in a child: a case report and review of the literature. J Med Case Rep. 2010; 4(1):234

7. Sood RK. Fractured tracheostomy tube. J Laryngol Otol. 1973; 87: $1033-4$

8. Brockhurst PJ, Feltoe CK. Corrosion and fracture of a silver tracheostomy tube. J Laryngol Otol. 1991; 105(01):48-9

9. Jensen OV, Pedersen U. Fractures in polyvinyl chloride tracheostomy tubes. J Laryngol Otol. 1988;102(04):380-1

10. Okafor BC. Fracture of tracheostomy tubes. Pathogenesis and prevention. J Laryngol Otol. 1983; 97(08):771-4

11. Krishnamurthy A, Vijayalakshmi R. Broken tracheostomy tube: A fractured mandate. J Emerg Trauma Shock. 2012; 5(1):97

12. So-ngern A, Boonsarngsuk V. Fractured metallic tracheostomy tube: A rare complication of tracheostomy. Respir Med Case Rep. 2016 ; 19:46-8

13. Karakoc F, Cakir E, Ersu R, Uyan ZS, Colak B, Karadag B, Kiyan G, Dagli T, Dagli E: Late diagnosis of foreign body aspiration in children with chronic respiratory symptoms. Int J Pediatr Otorhinolaryngol. 2007; 71: 241-246. 10.1016/j. ijporl.2006.10.006

14. Lynrah ZA, Goyal S, Goyal A, Lyngdoh NM, Shunyu NB, Baruah B, Dass R, Yunus M, Bhattacharyya P. Fractured tracheostomy tube as foreign body bronchus: our experience with three cases. Int J Pediatr Otorhinolaryngol. 2012; 76(11):1691-5

15. Tahir N, Ramsden WH, Stringer MD. Tracheobronchial anatomy and the distribution of inhaled foreign bodies in children. Eur J Pediatr. 2009; 168(3):289-95

16. Alqudehy ZA, Alnufaily YK. Fractured tracheostomy tube in the tracheobronchial tree of a child: Case report and literature review. J Otolaryngol Head Neck Surg. 2010; 39:E70-3

17. Ranjan K, Phookan J, Devi HR, Das MP. Broken Synthetic Tracheostomy Tube Presenting As Tracheobronchial Foreign Body-A Case Report. IOSR Journal of Dental and Medical Sciences (IOSR-JDMS);1(15):13-4

18. Loh TL, Chin R, Flynn P, Jayachandra S. Fracture and aspiration of a tracheostomy tube. BMJ Case Rep. 2014 Feb 19; 2014:bcr2013203232

19. Srirompotong S, Kraitrakul S: Fractured inner tracheostomy tube: an unusual tracheobronchial foreign body. Srinagarind Med J. 2001; 16: 223-5

20. Krempl GA, Otto RA: Fracture at fenestration of synthetic tracheostomy tube resulting in a tracheobronchial airway foreign body. South Med J. 1999; 92: 526-8. 